\title{
GM crop data-agronomy and ecology in tandem
}

\author{
C. Neal Stewart, Jr. and Sarah K. Wheaton
}

At a recent international gathering of biosafety researchers ${ }^{1}$, a European scientist overheard our American dialects and commented dryly, "I didn't think you Yanks cared about this sort of thing [biosafety]." There is, unfortunately, a grain of truth in that statement. However, we do care. And we argue here that biosafety data could be gathered efficiently if US researchers would collect ecological data during largescale trials and commercial uses of GM crops.

There is no evidence that the current products of GM crops produced in the United States are harmful to the environment or human health; an estimated 3.5 trillion GM plants grown in the United States since 1994 have had no measurable ill effects ${ }^{2}$. However, ecological interactions are complex, and the next generation of GM crops will have agronomic and output traits that are not as well understood as, say, those of Bacillus thuringiensis $(B t)$-derived GM products. For example, they will have multiple qualities enabling the plants to better cope with diseases, drought, toxic metals, as well as insects. Insect resistance genes will have a broader spectrum. Furthermore, the various enhanced traits may interact with one another to yield another layer of ecological complexity. To enhance our understanding of a particular GM crop/system in relevant ecological settings, we need risk assessment data from solidly designed ecological experiments. The data from such experiments are needed not only to satisfy regulatory requirements, but also to show what parameters need to be followed in post-commercialization monitoring.

The current GM crops conundrum is an indication that the current paradigm of risk assessment research in the United States may be askew. The US Department of Agriculture (USDA) allocates approximately $\$ 1.5$ million to peer-selected risk assessment research per year, a figure that translates into fewer than 10 standard projects. In contrast, the UK government last year allocated nearly 10 times as much in supporting various initiatives. The money has been invested in scaled-up ecological and agronomic experiments to assess the presence of possible real differences in the eco-

C. Neal Stewart, Jr. is at the University of North Carolina-Greensboro, Department of Biology, Greensboro, NC 27402

(nstewart@uncg.edu), and Sarah K. Wheaton is at the Graduate School of Oceanography, University of Rhode Island, South Ferry Road, Narragansett, RI 02882. logical performance of GM versus non-GM crops at the farm scale-where agriculture is practiced.

One can argue that understanding fully the impact of biotechnology on managed and unmanaged ecosystems is almost a global duty for the United States. The US agricultural biotechnology industry leads the world; the United States could and should also lead in biosafety research. In the United States, there is considerable freedom to test experimental GM plants in the field under the regulatory framework. European researchers, in contrast, must advertise the exact location of a GM crop experiment in their local newspapers, courting vandalism by militant antiGM activists. This relative security means that US biosafety research should be more efficient, given that the plants are more likely to be intact at the end of the experiment.

We want to encourage a new way of thinking about funding and performing GM crop ecological studies. At present, industry funds and performs field trials to examine transgene/variety agronomic performance, whereas academic researchers perform a few federally sponsored ecological projects. What would make more sense is to have ecological experiments performed on the same GM crops that will be later commercialized. Making this happen will require the combined public and private funds.

The same combined agronomic/ecological approach could also apply to deregulated and commercialized GM crops as they are deployed. Farmers could participate in sideby-side comparisons of GM and non-GM crops in similar settings. Although this approach may not provide the perfect ecological experimental design, it would provide honest data that could allay irrational fears. One study could be a community-level biodiversity survey of GM and non-GM fields to get a field-level grasp of ecological nontarget effects of pesticidal genes versus nontarget effects of non-GM crops and conventional chemical pesticide. A collaborative approach would increase the understanding and predictive accuracy of the performance of GM crops in agriculture and nature. Currently, many of the ecologist's biosafety studies use non-GM plants in an attempt to understand the dynamics of GM plants, an approach that lacks relevance and predictive power.

All parties would stand to benefit: industry in overcoming negative public perception and mistrust; the public and the regulators in obtaining additional and relevant data for cur- rent risk assessments and for future monitoring; and academics in acquiring funding. Certain companies, such as Aventis, are already performing post-commercialization monitoring in collaboration with academic agronomists, weed scientists, and entomologists. Such monitoring could be extended by bringing academic ecologists on board as well. Doing so would provide data and knowledge that could be very important for sustainability during ecological time scales (dozens of years).

There are several obstacles to implementing such combined research, but none is insurmountable. There is, for instance, mutual suspicion between industry and ecologists. The public is also wary of industryfunded academic science, encouraged in this, perhaps, by activists whose aim is to discredit the research. Such problems of trust could be mitigated by the decoupling of industry funding from academic research. We suggest that one solution might be to create a fund and GM crop pool that would be administered by the USDA or another federal agency. In an ideal world, perhaps, the USDA, the US Environmental Protection Agency, and the US Food and Drug Administration would jointly and seamlessly administer a pool of new money to support the interrelated and multidisciplinary study of ecological, food safety and exposure risks.

As with any collaborative $R \& D$ effort, there would be intellectual property and academic freedom considerations. But it shouldn't be beyond the wit of leaders from industry, academia, and government to establish rules of cooperative engagement that would, for instance, ensure timely publication of both favorable and unfavorable results while retaining sensitivity over intellectual property.

There is no real reason that the disparate agendas of the various parties should not crystallize around a common core. We believe that if combined agronomic and ecological studies had occurred more frequently in the past, current public perception of GM might be quite different, and the paranoia arising from a sense of being uninformed might be diminished.

\section{Acknowledgments}

Thanks for helpful comments and discussions with Fred Gould, Tom Nickson, Allison Snow, and especially Jeremy Sweet.

\footnotetext{
1. The National Research Council workshop. Ecological monitoring of transgenic crops. July 13-14, 2000 (Washington, DC).

2. Stewart, C.N. Jr., Richards, H.A. \& Halfhill, M.D. BioTechniques 29, 832-843 (2000).
} 